\title{
Les Mémoires policiers 1750-1850. Écritures et pratiques policières du Siècle des Lumières au Second Empire
}

Jean-Marc Berlière

\section{(2) OpenEdition \\ Journals}

Édition électronique

URL : https://journals.openedition.org/ahrf/9653

DOI : 10.4000/ahrf.9653

ISSN : 1952-403X

Éditeur :

Armand Colin, Société des études robespierristes

Édition imprimée

Date de publication : 1 juin 2007

Pagination : 230-233

ISSN : 0003-4436

\section{Référence électronique}

Jean-Marc Berlière, «Les Mémoires policiers 1750-1850. Écritures et pratiques policières du Siècle des Lumières au Second Empire », Annales historiques de la Révolution française [En ligne], 348 | AvrilJuin 2007, mis en ligne le 23 juillet 2008, consulté le 30 avril 2022. URL : http:// journals.openedition.org/ahrf/9653; DOI : https://doi.org/10.4000/ahrf.9653

Ce document a été généré automatiquement le 30 avril 2022.

Tous droits réservés 


\title{
Les Mémoires policiers 1750-1850. Écritures et pratiques policières $\mathrm{du}$ Siècle des Lumières au Second Empire
}

\author{
Jean-Marc Berlière
}

\section{RÉFÉRENCE}

Vincent Milliot (dir.), Les Mémoires policiers 1750-1850. Écritures et pratiques policières du Siècle des Lumières au Second Empire, Rennes, Presses Universitaires de Rennes-MRSH de Caen, 2006, 415 p., ISBN 2-9510796-1-3, $22 €$.

1 On doit à Vincent Milliot d'avoir pris à bras le corps le chantier si riche et prometteur des polices d'Ancien Régime, domaine resté trop longtemps une jachère de l'historiographie, en dépit de l'abondant usage fait par les modernistes des archives de police. De façon comparable à ce qui se passait pour l'histoire contemporaine il y a encore vingt ans, il restait à s'intéresser aux polices et aux policiers eux-mêmes. C'est désormais chose faite dans la suite logique des pistes proposées par Daniel Roche à la fin des années 1970. Avec ses complices habituels, Catherine Denys et Vincent Denis, rejoints par une pléiade de jeunes chercheurs, Vincent Milliot a coordonné une recherche plurielle sur une source d'une grande richesse et d'un intérêt considérable à laquelle il a déjà consacré un texte paru dans l'ouvrage dirigé par P. Laborier consacré aux Sciences camérales (PUF, 2006) : "Quand la police prend la plume: écritures et pratiques policières en France au siècle des Lumières ».

2 Les écrits policiers produits de 1750 à 1850, dont cet ouvrage rassemble des spécimens d'une grande variété, contribuent à mettre en lumière une zone d'ombre longtemps préjudiciable à la compréhension et à la connaissance que l'on pouvait avoir du monde 
des polices et des policiers sur la période qui s'étend, grosso modo, du Traité de police de Nicolas Delamare au deuxième tiers du XIX ${ }^{\mathrm{e}}$ siècle.

3 Si la fin du XIX ${ }^{\mathrm{e}}$ siècle a connu une inflation de l'écrit policier avec l'apparition de «mémoires »-au sens de souvenirs professionnels - écrits par des policiers profitant de l'intérêt grandissant du public pour le crime, l'énigme judiciaire, l'enquête, soutenu par le succès d'un genre littéraire qui s'épanouit à partir de Gaboriau, il n'en va pas de même pour la période qui précède. Ici point d'équivalent aux mémoires de ces «chefs de la Sûreté " comme Canler, Goron ou Macé évoquant les affaires criminelles auxquelles ils furent mêlés. Les " mémoires » auxquels le collectif d'auteurs réunis dans cet ouvrage s'intéresse, pour avoir majoritairement été écrits par des praticiens de la police, ressortissent à d'autres genres. Compilations de règlements, recueils de principes et de méthodes, manuels, vade-mecum, conseils, recettes et savoir-faire... ces textes, à usage interne, confidentiels, rarement confiés à l'édition, au caractère parfois revendicatif, transmettent une mémoire, des savoirs, mais prennent aussi la forme de projets ou de propositions de réformes qui soulignent les lignes de fractures des institutions et permettent de lire en négatif ce que sont durant toute cette période l'organisation des «polices » et les défauts qui les affectent. On notera qu'il s'agit là d'un genre appelé à durer et que l'on retrouve sous la IIIe République avec les essais de policiers, de parlementaires ou d'élus municipaux critiquant la police telle «qu'elle est » et s'attachant à définir « ce qu'elle devrait être » (E. Locard, Payot, 1919).

On imagine l'intérêt d'un tel corpus pour qui s'intéresse aux agents, aux forces en charge de ce que nous appelons par commodité «la» police, mais dont l'acception recoupe alors des fonctions très diverses selon la chronologie, les lieux, les corps et institutions concernés. Les auteurs de ces «mémoires»-dont l'hétérogénéité donne une bonne idée de la diversité des instances concurremment chargées de la sécurité publique et «du vivre ensemble »- donnent à voir un monde très divers, mêlant archaïsmes et novations. À travers leurs propos, se perçoit l'écho de débats passionnés sur la nature, les missions, les métiers de police, les hésitations entre modèles traditionnels et aspirations nouvelles, fondées sur des exemples étrangers ou les idéaux voire les utopies des Lumières : qu'on songe par exemple au Mémoire sur la réformation de la police... de l'exempt Guillauté (1749) qui, parmi d'autres novations, imagine un fichier central et une " machine » qui auraient comblé Michel Foucault.

5 Les différentes contributions apportent de nombreux éclairages particulièrement bienvenus. La singularité et l'exceptionnalité du modèle parisien qu'avaient déjà soulignées les travaux de Catherine Denys et J.-L. Laffont se trouvent ici confirmées par les cas de Lyon (S. Nivet), Strasbourg et Bordeaux (V. Denis), Grenoble (C. Coulomb), Nantes (S. Perréon), Lille (C. Denys) tandis que P. Brouillet illustre le rôle pionnier de la maréchaussée dans la construction de savoir-faire professionnels, le souci de leur transmission et de la formation nécessaire des cavaliers.

6 Judicieusement articulé en trois thèmes - écrire pour améliorer, écrire pour réformer, écrire pour refonder la police - présentés avec beaucoup de clarté dans des synthèses introductives qui rappellent sans cesse les enjeux et permettent de suivre chronologie et problématiques, l'ouvrage quoique collectif conserve une réelle homogénéité. Il la doit en particulier à la récurrence et à la déclinaison de thèmes et questionnements essentiels que l'on retrouve peu ou prou dans la plupart des « mémoires » étudiés et qui tournent autour des pouvoirs de police, de leur exercice et de leur organisation, des 
savoirs et de leur apprentissage, de la professionnalisation, de la rétribution, de la formation des agents en charge des missions de sûreté publique.

7 Cette «littérature policière " protéiforme qui s'inscrit souvent dans des stratégies personnelles et en dit parfois aussi long sur ses destinataires que sur ses auteurs, permet d'interroger la nature même de «la " police, ses moyens, ses ambitions, ses illusions, les méthodes qu'elle doit mettre en œuvre : en bref ce que policer veut dire... à la notable exception - tant le secret semble gage d'efficacité - de ces pratiques de l'ombre qui caractérisent la police parisienne depuis d'Argenson.

On y lit également le souci récurrent de l'ordre et de son maintien, la volonté d'adaptabilité et de réactivité tout comme la quête d'un idéal de police que les auteurs de ces « mémoires » tentent de dessiner et de faire partager dans un dialogue virtuel et intemporel mené via des textes qui se répondent, s'opposent, se complètent dans un débat jamais achevé et indéfiniment relancé par les conflits et les enjeux nés des spécialisations et concurrences de compétences qui caractérisent ce champ...

9 L'historien de la police contemporaine ne peut qu'être frappé des continuités que ces textes démontrent et il découvre les racines lointaines de politiques publiques qui caractériseront la IIIe République. Exemplaire à cet égard est le mémoire du major de la Garde de Paris Jean-François de Bar (1772) étudié par P. Péveri qui aborde le problème de la technique du maintien de l'ordre à Paris face à l'émeute. Outre la mise en œuvre de tactiques (avec schémas explicatifs) qu'il n'aurait pas reniées, le préfet Lépine y aurait découvert une volonté de "se faire aimer en remplissant ses devoirs" et d'acquérir ainsi des «droits réels à l'estime publique » qui fut aussi l'une de ses obsessions : faire aimer la police pour la rendre plus efficace.

10 Si l'Ancien Régime gagne beaucoup à ces études très neuves, le premier $\mathrm{XIX}^{\mathrm{e}}$ siècle n'est pas en reste. L'un des apports de l'ouvrage est de montrer un souci permanent de relégitimer et penser une police et une gendarmerie en évitant «le despotisme de la monarchie, la folie terroriste et inquisitoriale des révolutionnaires, les pratiques liberticides de l'Empire » : vaste programme qui va constituer le cœur de la réflexion sur la police tout au long du XIX ${ }^{e}$ siècle pour aboutir (?) à la veille de la Première guerre mondiale à un «modèle républicain » bien fragile qui doit finalement beaucoup à la " police philosophique » à laquelle aspirait Manuel et que plusieurs mémoires tentent de définir ou d'ébaucher avec une mention toute spéciale à la réflexion de Sieyès sur le maintien de l'ordre. On y découvre un souci de graduer et d'adapter l'usage d'une force publique que l'on cherche à différer le plus tard possible qui constitue l'un des principes de la doctrine du maintien de l'ordre contemporain théorisé par une « instruction » de la gendarmerie mobile datant de... 1930.

11 Ces quelques réflexions sont bien loin d'épuiser les richesses d'un ouvrage dont on saluera également les auteurs pour l'initiative d'avoir publié plusieurs des mémoires cités tant il est vrai que l'histoire se nourrit d'archives et que mettre celles-ci (par nature dispersées, difficiles d'accès...) à la disposition du public est non seulement une des missions de l'historien, mais également le moyen de donner à chaque lecteur la possibilité de lire la totalité des textes, d'en rapprocher la lettre de l'étude qui en est faite: un souci de transparence et de pédagogie dont on doit louer également un éditeur qui confirme sa place et son importance dans le champ historique (on devait déjà aux PUR, l'édition, en 2001, des travaux d'un premier colloque "transversal »: Police et migrants en France 1667-1939 sous la direction de M.-C. Blanc-Chaléard, C. Douki, N. Dyonet et V. Milliot). 
12 Cet ouvrage marque donc une étape importante dans un chantier qui, après une trop longue inertie, est désormais en pleine activité comme l'ont montré ces derniers mois la journée d'étude consacrée aux commissaires de police au XIX $\mathrm{X}^{\mathrm{e}}$ siècle (la publication des actes est prévue pour la fin 2007 aux Presses de la Sorbonne) et le colloque « Être policier en Europe $18^{\mathrm{e}}-20^{\mathrm{e}}$ siècles » qui vient de se tenir à Caen et qui a notamment permis de découvrir les avancées importantes d'une histoire de la police qui gagne des pays comme la Grèce, la Roumanie, l'Empire Ottoman qui figuraient jusqu'à présent en blanc dans la cartographie d'une histoire en plein essor. 